\title{
Orientation of Single Dibenzanthanthrene Molecules in Solid Xenon
}

\author{
J. Sepiǫ*, R. KoŁos And J. Jasny \\ Institute of Physical Chemistry, Polish Academy of Sciences \\ Kasprzaka 44/52, 01-224 Warsaw, Poland \\ Dedicated to the memory of Professor Jerzy Prochorow
}

\begin{abstract}
The spatial distributions of individual dibenzanthanthrene electronic transition dipole moments in solid Xe was investigated at the single molecule level, with the high-resolution fluorescence spectroscopy. Samples were prepared by the co-deposition of dibenzanthanthrene/Xe vapours onto the $50 \mathrm{~K}$ surface. The results indicate that the orientation of planar aromatic molecules is preferentially parallel to the trapping surface.
\end{abstract}

PACS numbers: 33.50.-j, 61.66.Bi, 61.66.Hq. 61.72.Ww

\section{Introduction}

Dibenzanthanthrene (DBATT) is a useful fluorophore, extensively studied in low temperature matrices at the single molecule level. Photophysical parameters deduced from single molecule experiments in the hexadecane Shpol'skii matrix pointed to a very low population of the DBATT triplet state [1]. Due to the high photostability, and to the absence of spectral diffusion for DBATT embedded in naphthalene crystals, both Stark effect measurements, and interesting pump-probe experiments with strong laser fields could be performed for single molecules $[2,3]$. Microscopic polarization studies on DBATT in $n$-tetradecane revealed that guest molecules may exhibit, in a specific site of the Shpol'skii matrix, the long-range order over distances as large as approximately $2 \mathrm{~mm}$ [4]. This interesting result contradicts the common concept of Shpol'skii systems as highly disordered ones. Recent low temperature studies on DBATT in spincoated high-density polyethylene (HDPE) films $[5,6]$ showed the strong orientation preference of probe molecules [6], and the lack of such preference for low-density PE (LDPE).

${ }^{*}$ corresponding author; e-mail: sepiol@ichf.edu.pl 
We have demonstrated in earlier reports $[7,8]$ that fluorescence from single DBATT molecules could also be observed in rare gas matrices, namely in krypton and in xenon. The temperature behaviour was found to be very dissimilar for different DBATT molecules observed in a solid Xe matrix, and was reflected in spectral widths of measured zero-phonon lines for the electronic transition [8]. Taking advantage from the application of a microscopy objective developed specially for cryogenic measurements, and allowing for the study of single molecules and quantum dots $[9,10]$, we undertook a study on the orientation of DBATT molecules co-deposited with Xe atoms on a cold surface.

\section{Experimental}

The mirror microscopy objective [9] used in this study featured the annular aperture of 0.722 and a magnification of about 100. The sample of gaseous xenon, mixed with DBATT (PAH Research Institute, Greifenberg) vapour from a ca. $550 \mathrm{~K}$ oven, was solidified directly onto the quartz objective surface, as described earlier [8]. The cryostat cold finger, holding the objective, was kept during the deposition at $50 \mathrm{~K}$, allowing for the formation of a "relaxed" solid, the latter quality being important for the generation of stable fluorescence signals from single molecules after the sample cool-down to about $2 \mathrm{~K}$.

In order to measure fluorescence excitation spectra of DBATT in solid xenon, a broad band cw dye laser, tunable by Lyott filters (band width of $20 \mathrm{GHz}$ ) was employed. Fluorescence produced by illuminated molecules, filtered by an appropriate Schott glass (supressing the scattered laser beam), was measured with a photomultiplier.

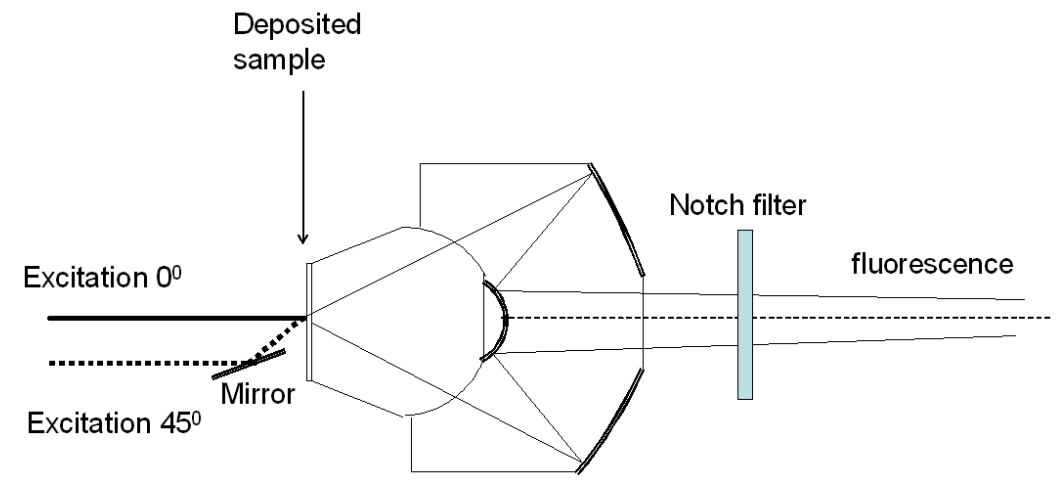

Fig. 1. Laser beam configurations for two modes of sample excitation, referred to as $0^{\circ}$ (solid line) and $45^{\circ}$ (dotted line) in the text.

A stabilized cw single-mode dye laser (Coherent 899) operated with rhodamine-6G [11] was used for the selective excitation of single molecules. To learn more about the orientations of transition dipole moments for individual 
molecules, the laser radiation was split into two beams of similar intensities, which illuminated the sample, as seen in Fig. 1, at two different angles of incidence: $0^{\circ}$ (with the electric vector normal to the objective optical axis), and $45^{\circ}$. A CCD camera, model 4Quick $\Phi 5 \mathrm{~A}$, cooled by the Peltier elements, registered the patterns of fluorescing spots (separated from the scattered light by a Notch filter), both in the objective focal plane, and several centimeters out of the plane. The spatial distribution of the fluorescence emitted by a single molecule, recorded out of the focal plane, was analysed, similarly as in Ref. [12], by a home-written program, which yielded radial and azimuthal orientation angles of the transition dipole moment.

\section{Results and discussion}

The fluorescence excitation spectrum of DBATT, co-deposited with Xe atoms on the entrance surface of the microscope objective, is presented in Fig. 2. The shapes and positions of two bands, marked by letters A and B, match the absorption bands measured in solid Xe [8]. The band A, with a maximum around $17075 \mathrm{~cm}^{-1}$, is structured, broader than $\mathrm{B}$, and reveals the contributions from several DBATT/Xe sub-sites. Feature B, around $17155 \mathrm{~cm}^{-1}$, originates rather in a single matrix site.

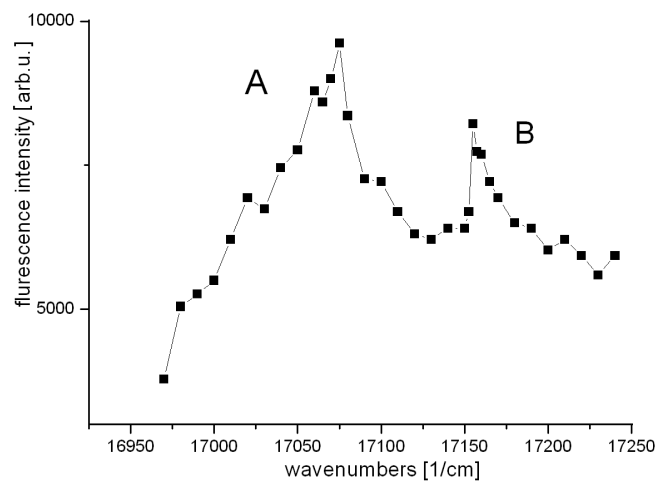

Fig. 2. Broadband DBATT fluorescence excitation spectrum, as measured in solid Xe at $2 \mathrm{~K}$.

The observation of individual DBATT molecules in our experiments was facilitated by the well-known "spectral selection" phenomenon, characteristic of solids at low temperatures [13]. It consists in the spectral narrowing of the 0-0 electronic transition, down to a lifetime-limited spectral width. In the case of DBATT, where the fluorescence lifetime is $10 \mathrm{~ns}$ [8], the lower limit of the 0-0 transition spectral width is about $20 \mathrm{MHz}$. By adapting the cw broad-band dye laser to operate in the single-mode regime $(2-3 \mathrm{MHz})$, one can selectively excite only those molecules, which happen to be in resonance with a chosen laser wavelength. 
It should be noticed that the excitation with linearly (or circularly) polarized laser beam, oriented along the optical axis of the objective (excitation $0^{\circ}$; Fig. 1 ), leads to the efficient excitation of those molecules, for which the electronic transition moment lies in the plane of the sample. To get insight to the real distribution of individual transition dipole moments, we made use of two excitation angles: $0^{\circ}$ and $45^{\circ}$. Both geometrical configurations led, for excitation wavelengths corresponding to bands $\mathrm{A}$ and $\mathrm{B}$ (Fig. 2), to the detection of bright spots in the objective focal plane, indicative of single fluorescing DBATT molecules. The variations of intensity, from one spot to another, resulted mostly from the fact that only a fraction of molecules was excited optimally, i.e. at the maximum of the $0-0$ absorption band. Figure 3 presents the typical pair of images, corresponding to $0^{\circ}$ and $45^{\circ}$ excitations. The analysis of such pairs led to the conclusion that $45^{\circ} \mathrm{im}-$ ages differ from those recorded for $0^{\circ}$ only by the relative brightness of individual spots. In particular, the $45^{\circ}$ excitation did not reveal any "new" objects, i.e. not present at $0^{\circ}$; this suggests a strong preference for such orientations of DBATT molecules in $\mathrm{Xe}$, for which the value of the transition dipole moment component perpendicular to the optical axis is high.

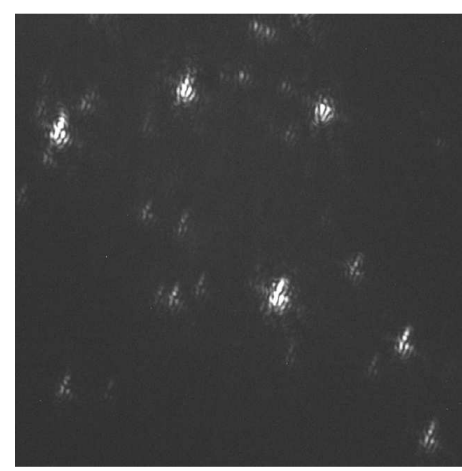

Excitation $0^{\circ}$

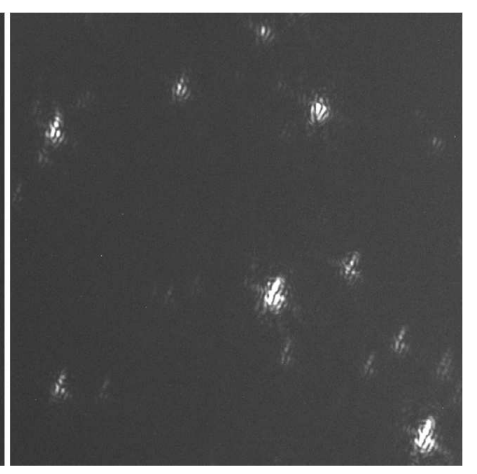

Excitation $45^{\circ}$

Fig. 3. Typical pair of microscopic images differing by the configuration of the circularly polarised laser beam.

As demonstrated in an earlier paper [12], dealing with terrylene in $n$-dodecane, the analysis of luminous pattern from a single molecule, detected out of the focal plane, gives the full information necessary for the assessment of the transition moment orientation (radial and azimuthal angles). The same technique has been used in the present work. Its weak point consists in the ambiguity inherent to the theoretical fitting, stemming from the similarity of fluorescence distributions for small and large radial angles (i.e. angles between the transition moment vector and the optical axis). Rather than just one radial angle for a given molecule, the analysis may in fact give two, as illustrated by the following pairs of numbers, obtained for a set of single molecules in one of our experiments: $88^{\circ}-0^{\circ}$, 
$83^{\circ}-1^{\circ}, 75^{\circ}-3^{\circ}, 66^{\circ}-4^{\circ}, 62^{\circ}-5^{\circ}, 43^{\circ}-9^{\circ}, 40^{\circ}-10^{\circ}, 35^{\circ}-11^{\circ}$. This uncertainty can be removed by an additional measurement, with the laser excitation beam inclined out of the optical axis. In our case, the measurements done at excitation angles $0^{\circ}$ and $45^{\circ}$ pointed to a simple scheme: the larger of two numbers should be taken as the real radial angle value.

Figures $4 \mathrm{~A}$ and $4 \mathrm{~B}$ depict the histograms obtained for azimuthal ( $\phi$; measured relatively to an arbitrarily chosen horizontal direction) and radial $(\Delta)$ angles, describing spatial orientations of 22 molecules excited within the range of band $\mathrm{A}$. Noteworthy, no preference for any particular values of $\phi$ was found. Conversely, the radial angles were distributed preferentially in the $30^{\circ}-90^{\circ}$ range.

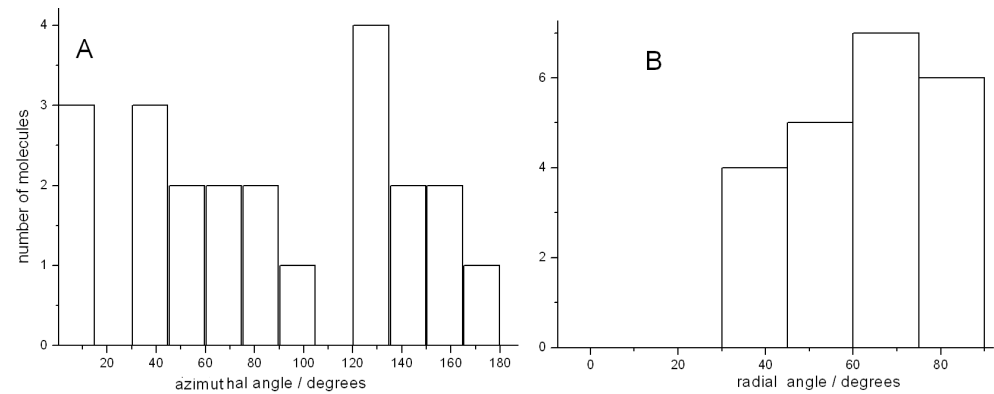

Fig. 4. Distribution of the azimuthal (A) and radial (B) angles defined for the transition dipole moment, as measured for 22 DBATT molecules in solid Xe at $2 \mathrm{~K}$.

These results come as no surprise, given that the transition dipole moment of DBATT, for the long-wavelength absorption, is confined within the molecular plane, parallel to the long axis of this polycyclic aromatic hydrocarbon [14]. The condensation of xenon atoms at a relatively high temperature of $50 \mathrm{~K}$ permits for the close packing, and thus for the formation of a layered structure. DBATT molecules, occasionally hitting such a surface, are attracted to it with van der Waals forces. Eventually, a homogeneous distribution of azimuthal angles $(\phi)$ is to be expected, but the radial angles $(\Delta)$ should be large. The degree of respective order can be evaluated from the radial angles distribution. In fact, the dispersion of $\Delta$ angles proves to be quite large: more than $80 \%$ of molecules had $\Delta$ confined within the range $45^{\circ}-90^{\circ}$, and large values of $75^{\circ}-90^{\circ}$ were found for only $30 \%$ of analyzed objects.

For molecules absorbing within the narrow range of band B (blue-shifted, with respect to A, by $c a .80 \mathrm{~cm}^{-1}$ ), the distributions of angles $\phi$ and $\Delta$ were similar to those in band A, shown in Fig. 4. However, the assignment of B to a specific DBATT site in xenon is questionable, in the context of fluorescence excitation spectra obtained for Shpol'skii matrices. For instance, in the tetradecane matrix, a "site 2" band was reported about $70 \mathrm{~cm}^{-1}$ to the blue of the main "site 1 " [4]. Such similar spectroscopies in completely different hosts speak against the attribution of both bands to matrix sites. An alternative explanation of these 
results involves the hypothesis that studied DBATT samples (whose origin in all published works is the same) are contaminated with another fluorescing compound. This point deserves analyses by complementary techniques.

Summing up, the above reported spatial distributions of the fluorescence from single DBATT molecules allowed us to reveal their preferred orientation in solid Xe: respective radial angles $(\Delta)$ of transition moments tend to cluster within the range $45^{\circ}-90^{\circ}$.

\section{Acknowledgments}

The authors are grateful to Prof. Urs P. Wild for the introduction to the field of single molecule fluorescence spectroscopy, support in Zurich and stimulating discussions. We are indebted to Dr. A. Renn for his valuable help in setting up the experiment at ETH, and to Mr. Guan Yinghua for the assistance during measurements.

\section{References}

[1] A.-M. Boiron, B. Lounis, M. Orrit, J. Chem. Phys. 105, 3969 (1996).

[2] Ch. Brunel, Ph. Tamarat, B. Lounis, J.C. Woehl, M. Orrit, J. Phys. Chem. A 103, 2429 (1999).

[3] F. Jelezko, B. Lounis, M. Orrit, J. Chem. Phys. 107, 1692 (1997).

[4] A. Bloess, Y. Durand, M. Matsushita, R. Verberk, E.J.J. Groenen, J. Schmidt, J. Phys. Chem. A 105, 3016 (2001).

[5] A.C. Wirtz, M. Dokter, C. Hofmann, E.J.J. Groenen, Chem. Phys. Lett. 417, 383 (2006).

[6] A.C. Wirtz, C. Hofmann, E.J.J. Groenen, J. Phys. Chem. B 110, 21623 (2006).

[7] J. Sepioł, A. Starukhin, R. Kołos, T. Latychevskaia, J. Jasny, A. Renn, U.P. Wild, Chem. Phys. Lett. 311, 29 (1999).

[8] J. Sepioł, R. Kołos, A. Renn, U.P. Wild, Chem. Phys. Lett. 355, 71 (2002).

[9] J. Jasny, J. Sepioł, T. Irngartinger, M. Traber, A. Renn, U.P. Wild, Rev. Sci. Instrum. 67, 1425 (1996).

[10] A. Kudelski, K. Kowalik, A. Golnik, G. Karczewski, J. Kossut, J.A. Gaj, J. Lumin. 112, 127 (2005).

[11] T. Nonn, T. Plakhotnik, Chem. Phys. Lett. 336, 97 (2001).

[12] J. Sepioł, J. Jasny, J. Keller, U.P. Wild, Chem. Phys. Lett. 273, 444 (1997).

[13] Single Molecule Optical Detection, Imaging and Spectroscopy, Eds. T. Basche, W.E. Moerner, M. Orrit, U.P. Wild, Verlag-Chemie, Weinheim 1997.

[14] J. Sepioł, unpublished results. 